\title{
XXXVII. A general formula for the moments of the normal correlation function of any number of variates
}

\section{S.D. Wicksell}

To cite this article: S.D. Wicksell (1919) XXXVII. A general formula for the moments of the normal correlation function of any number of variates, Philosophical Magazine Series 6 , 37:220, 446-452, DOI: $10.1080 / 14786440408635900$

To link to this article: http://dx.doi.org/10.1080/14786440408635900

曲 Published online: 08 Apr 2009.

Submit your article to this journal $₫$

Џll Article views: 3

Q View related articles ๘ 
The experiments of Franck and Hertz show that in some cases, for example, argon and nitrogen, an electron may collide with a very large number of atums without losing energy, and in cases where the electrons cannot make many collisions before being captured and attached to an atom, the effects seem to be reconcilable with the view that such collisions as occur are elastic, and that the alternatives are capture or escape with undiminished energy.

Since the frequency of vibrations of an electron is proportional to the magnetic force it is evident that it would be affected by an external magnetic field; it would also be affected by an external electric field, since this would displace the position of equilibrium. The consideration of these effects must, however, be left for another occasion.

XXXVII. A General Formula for the Moments of the Normal Correlation Function of any Number of Variates. By S. D. Wicrsell, Lund, Sweden *

IF $x_{1}, x_{2}, \ldots x_{n}$ be $n$ variates reckoned from their 1 respective means and expressed in their respective dispersions as units, and $r_{p q}$ is the coefficient of correlation of $x_{p}$ and $x_{q}$, the normal correlation function is given by

$$
\phi\left(x_{1}, x_{2}, \ldots x_{n}\right)=\frac{1}{(2 \pi)^{n / 2} \sqrt{\bar{S}}} e^{-\frac{1}{2 S} \Sigma \Sigma S_{p q} x_{p} x_{q}}, \quad .
$$

where

and

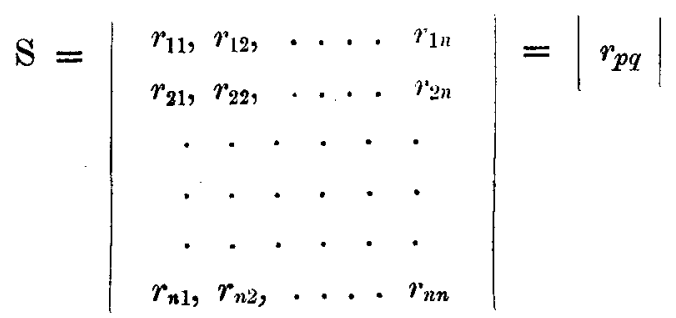

$$
\mathrm{S}_{p q}=\frac{\partial \mathrm{S}}{\partial r_{p q}} \text {. }
$$

The function $\phi\left(x_{1}, x_{2}, \ldots x_{n}\right)$ may also be written in the form of a certain multiple integral which will be

* Communicated by the Author. 
found to be very useful in discussing the mathematical properties of the function. Indeed we have

$$
\begin{aligned}
\phi\left(x_{1}, x_{2}, \ldots x_{n}\right)=\frac{1}{(2 \pi)^{n}} & \int_{-\infty}^{\infty} d w_{1} \int_{-\infty}^{\infty} d w_{2} \ldots \\
& \int_{-\infty}^{\infty} d w_{n} e^{-\frac{1}{2} \Sigma \Sigma r_{p 1} w_{p} w_{q}} e^{-i \Sigma x_{p} u_{p}}
\end{aligned}
$$

This integral, which for $n=1$ reduces to the well-known integral of Laplace, has been given by Charlier for the special case of $n=2$. A proof of the theorem for any value of $n$ will be given in the Appendix.

By partial integration it is further easily verified that

$$
\begin{aligned}
& k_{1}+k_{2}+\ldots+k_{n} x_{1}^{k_{1}} x_{2}^{k_{2}} \ldots x_{n}^{k_{n}} \phi\left(x_{1}, x_{2}, \ldots \ldots x_{n}\right)= \\
& =\frac{1}{(2 \pi)^{n}} \int_{-\infty}^{\infty} d w_{1} \int_{-\infty}^{\infty} d w_{2} \ldots \int_{-\infty}^{\infty} d w_{n} \frac{\partial^{k_{1}+k_{2}}+\ldots k_{n} e^{-\frac{1}{2} \Sigma \Sigma r_{p q} w_{p} w_{q}}}{\partial w_{1}^{k_{1}} \partial w_{2}^{k_{2}} \ldots \partial w_{n}^{k_{n}}}-i \Sigma x_{r} n^{k_{p} p}
\end{aligned}
$$

Hence by the theorem of Fourier we have

$$
\begin{gathered}
i^{k_{1}+k_{1}+\ldots+k_{n}} \int_{-\infty}^{\infty} d x_{1} \int_{-\infty}^{\infty} d x_{2} \ldots \int_{-\infty}^{\infty} d x_{n 2} x_{1}^{k_{1}} x_{2}^{k_{2}} \ldots x_{n}^{k_{n}} \phi\left(x_{1}, x_{s}, \ldots x_{n}\right)= \\
=\left[\frac{\partial^{k_{1}+k_{2}+\ldots+k_{n}} e^{-\frac{1}{2} \Sigma \Sigma} r_{p q} w_{p} w_{q}}{\partial w_{1}^{k_{1}} \partial w_{2}^{k_{2}} \ldots \partial w_{n}^{k_{n}}}\right]_{w_{1}=w_{2}=\ldots .=u_{n}=0^{\circ}}
\end{gathered}
$$

Putting

$$
\frac{\partial^{k_{1}+k_{2}+\ldots k_{n}} e^{-\frac{1}{2} \Sigma \Sigma r_{p q} w_{p} w_{q}}}{\partial w_{1}^{k_{1}} \partial w_{2}^{k_{2}} \ldots \partial \partial w_{n}^{k_{n}}}=\mathrm{H}_{k_{1} k_{2} \ldots k_{n}}^{\left(w_{1}, w_{2}, \ldots w_{n}\right)} e^{-\frac{1}{2} \Sigma \Sigma r_{p q} w_{p} w_{q}}
$$

we find, as

$$
\mathrm{H}_{k_{1} k_{2} \ldots, \ldots \dot{k}_{n}}(0,0)=0
$$

when $k_{1}+k_{2}+\ldots+k_{n}$ is an odd number, for the moments of any order of $\phi\left(x_{1}, x_{2}, \ldots x_{n}\right)$ the formula

$$
\begin{array}{r}
\int_{-\infty}^{\infty} d x_{1} \int_{-\infty}^{\infty} d x_{2} \ldots \int_{-\infty}^{\infty} d x_{n} x_{1}^{k_{1}} x_{2}^{k_{2}} \ldots \ldots x_{n}^{k_{n}} \phi\left(x_{1}, x_{2}, \ldots x_{n}\right) \\
=(-1) \frac{k_{1}+k_{2}+\ldots+k_{n}}{2} \\
H_{k_{1} k_{2} \ldots \ldots k_{n}}(0,0, \ldots 0)
\end{array}
$$


448 Prof. S. D. Wicksell on the Normal Correlation

We have now, remembering that in the case under consideration $r_{p q}=r_{q p}$ and $r_{p p}=1$,

$$
\begin{aligned}
& \mathbf{H}_{200 \ldots 0}=\left[\Sigma r_{1 q} v_{q}\right]^{2}-1 \text {, } \\
& \mathrm{H}_{110 \ldots 0}=\Sigma r_{1 q} w_{q} \Sigma r_{2 q} w_{q}-r_{12} \text {, } \\
& \mathrm{H}_{400 \ldots 0}=\left[\Sigma r_{1 q} w_{q}\right]^{4}-6\left[\Sigma r_{1 q} w_{q}\right]^{2}+3 \text {, } \\
& \mathbf{H}_{310 \ldots 0}=\left[\Sigma_{r_{1}{ }_{1} w_{q}}\right]^{3} \cdot \Sigma_{r_{2} w_{q}}-3 \Sigma_{1} r_{1} w_{q} \cdot \Sigma r_{2_{q}} w_{q} \\
& -3\left[\Sigma r_{1 q} w_{q}\right]^{2} r_{12}+3 r_{12} \text {, } \\
& \mathrm{H}_{220 \ldots 0}=\left[\Sigma r_{1 q} w_{q}\right]^{2}\left[\Sigma r_{2 q} w_{q}\right]^{2}-\left[\Sigma r_{2 q} w_{q}\right]^{2}-\left[\Sigma r_{1 q} w_{q}\right]^{2} \\
& -4 r_{12} \Sigma r_{1 q} w_{q} . \Sigma r_{2 q} w_{q}+1+2 r_{12}{ }^{2} \\
& \mathrm{H}_{2110 \ldots 0}=\left[\Sigma r_{1 q} w_{q}\right]^{2} \Sigma r_{2 q} w_{q} \cdot \Sigma r_{3 q} w_{q}-\Sigma r_{2 q} w_{q} \Sigma r_{3 q} w_{q} \\
& -2 r_{12} \Sigma r_{1 q} u_{q} \Sigma r_{3 q} w_{q}-2 r_{13} \Sigma r_{1 q} w_{q} \Sigma r_{2 q} w_{q} \\
& -r_{23}\left[\Sigma r_{1 q} v_{q}\right]^{2}+r_{23}+2 r_{21} r_{13} \\
& \mathrm{H}_{11110 \ldots 0}=\Sigma r_{1 q} w_{q} \cdot \Sigma r_{2 q} w_{q} \cdot \Sigma_{r_{3 q} w_{q}} \Sigma r_{4 q} w_{q} \\
& -r_{12} \Sigma r_{3 q} w_{q} \Sigma r_{4 q} w_{q}-r_{13} \Sigma r_{2 q} w_{q} \Sigma r_{4 q} w_{q} \\
& -r_{23} \Sigma r_{1 q} w_{q} \Sigma r_{4 q} w_{q}-r_{14} \Sigma r_{2 q} w_{q} \Sigma r_{3 q} w_{q} \\
& -r_{24} \Sigma r_{1 q} w_{q} \Sigma r_{3 q} w_{q}-r_{34} \Sigma r_{1 q} u_{q} \Sigma r_{2 q} w_{q} \\
& +r_{12} r_{34}+r_{13} r_{24}+r_{23} r_{14} \text {. } \\
& \int_{-\infty}^{\infty} d x_{1} \int_{-\infty}^{\infty} d x_{2} \ldots \int_{-\infty}^{\infty} d x_{n} x_{1}^{k_{1}} x_{2}^{k_{2}} \ldots \ldots x_{n}^{k_{n}} \phi\left(x_{1}, x_{2}, \ldots x_{n}\right)=m_{k_{1} k_{2} \ldots k_{n}},
\end{aligned}
$$

we find consequently

$$
\begin{aligned}
& m_{20} \ldots 0=1 \\
& m_{110} \ldots 0=r_{12}, \\
& m_{40} \ldots 0=3 \\
& m_{310} \ldots 0=3 r_{12}, \\
& m_{220} \ldots 0=1+2 r_{12}^{2}, \\
& m_{2110} \ldots 0=r_{23}-2 r_{12} r_{13}, \\
& m_{11110 \ldots 0}=r_{12} r_{34}+r_{13} r_{24}+r_{23} r_{14}
\end{aligned}
$$

and any other moment of the second and fourth orders may be obtained by permutation of indices.

Without deducing the polynoms $H$ of the sixth order, the moments of the sixth order may be determined in the following way :-

Evidently we have

$$
\mathrm{H}_{k_{1} k_{2} \ldots k_{s}+1 \ldots k_{n}}=\frac{\partial \mathrm{H}_{k_{2}, k_{2} \ldots \ldots k_{n}}}{\partial w_{s}}-\sum r_{s q} w_{q} \mathrm{H}_{k_{1} k_{2} \ldots k^{n}},
$$


Function of any Number of Variates.

Using this formula twice, we find, putting

$$
w_{1}=w_{2}=\ldots=w_{\mathfrak{n}}=0
$$

$$
\begin{aligned}
& H_{2}(0,0, \ldots, 0) \\
& k_{1} k_{2} \ldots k_{s}+1 \ldots k_{t+1} \ldots . k_{n} \\
& =r_{s t} H_{k_{2} k_{2}, \ldots k_{n}}(0,0, \ldots 0)-(-1)^{\frac{k_{1}+k_{2}+\ldots k_{n}}{2}}\left[\frac{\partial^{2} \mathrm{H}_{k_{2}} k_{2} \ldots k_{n}}{\partial w_{s} \partial w_{t}}\right]_{w_{2}=w_{2}=\ldots=w_{n}=0} ; \\
& \text { or } \\
& m_{k_{1} k_{2}} \ldots k_{s}+2 \ldots k_{n} \\
& =m_{k_{1}+k_{2}} \ldots k_{n_{n}}-(-1)^{\frac{k_{1}+k_{2}+\ldots k}{2}}\left[\frac{\partial^{2} \mathrm{H}_{k_{1} k_{2^{2}} \ldots k_{n}}}{\partial w_{s}^{2}}\right]_{w_{1}=w_{2}=\ldots \ldots=w_{n}=0} \text {, } \\
& m_{k_{1}} k_{2} \ldots k_{s}+1 \ldots k_{t}+1 \ldots k_{n} \\
& \text { 1) }\left[\frac{k_{1}+k_{2}+\ldots .+k_{n}}{2}\left[\frac{\partial^{2} H_{k_{1} k_{2} \ldots k_{n}}}{\partial w_{s} \partial w_{t}}\right]_{w_{1}=w_{2}=\ldots w_{n}=0^{\circ}}\right]
\end{aligned}
$$

Hence we find, with the aid of formula (5) and (6),

$$
\begin{aligned}
& m_{60} \ldots .00=15 \\
& m_{510} \ldots 0=15 r_{12} \text {, } \\
& m_{420} \ldots \ldots=3+12 r_{12}{ }^{2} \text {, } \\
& m_{4110} \ldots 0=3 r_{23}+12 r_{12} r_{13} \text {, } \\
& m_{330} \ldots . n=9 r_{12}+6 r_{18}{ }^{3} \text {, } \\
& m_{3210} \ldots 0=3 r_{13}+6 r_{12} r_{23}+6 r_{12}{ }^{2} r_{13} \\
& m_{31110} \ldots 0=3 r_{13} r_{24}+3 r_{12} r_{34}+3 r_{28} r_{14}+6 r_{12} r_{14} r_{13} \text {, } \\
& m_{2220} \ldots .=1+2 r_{12}{ }^{2}+2 r_{13}{ }^{2}+2 r_{23}{ }^{2}+8 r_{12} r_{13} r_{23} \text {, } \\
& m_{22110} \ldots 0=r_{34}+2 r_{13} r_{14}+2 r_{23} r_{24}+2 r_{12}^{2} r_{34}+4 r_{12} r_{13} r_{24} \\
& +4 r_{12} r_{14} r_{23} \\
& m_{21110 \ldots 0} \ldots=r_{23} r_{45}+r_{24} r_{35}+r_{25} r_{34}+2 r_{12} r_{13} r_{45}+2 r_{23} r_{14} r_{10} \\
& +2 r_{13} r_{14} r_{25}+2 r_{13} r_{15} r_{24}+2 r_{12} r_{14} r_{35}+2 r_{12} r_{15} r_{34} \\
& m_{111110 \ldots} \ldots=r_{23} r_{14} r_{56}+r_{13} r_{24} r_{66}+r_{12} r_{34} r_{56}+r_{34} r_{15} r_{26}+r_{34} r_{16} r_{25} \\
& +r_{24} r_{15} r_{36}+r_{24} r_{16} r_{35}+r_{14} r_{25} r_{36}+r_{14} r_{26} r_{35} \\
& +r_{23} r_{16} r_{46}+r_{23} r_{16} r_{45}+r_{13} r_{25} r_{46}+r_{13} r_{26} r_{45} \\
& +r_{12} r_{35} r_{46}+r_{12} r_{36} r_{45} \text {, }
\end{aligned}
$$

and any other moment of the sixth order is ottained by permutation of indices.

Phil. Mag. S. 6. Vol. 37 No. 220. April 1919. 2 I 
The moments of the variates expressed in any units being denoted by $\nu_{k_{1} k_{2} \ldots k_{n}}$, we have further

$$
\nu k_{1} k_{2} \ldots k_{n}=\sigma_{1}{ }^{k_{1}} \sigma_{2}{ }^{k_{2}} \ldots \sigma_{n}^{k_{n}} m_{k_{1} k_{2}} \ldots k_{n},
$$

where $\sigma_{1}, \sigma_{2}, \ldots \sigma_{n}$ are the respactive dispersions of the variates.

On account of the reciprocity of the determinants $S$ and $\Delta$ we have finally, putting

$$
\frac{\mathbf{S}_{p q}}{\mathbf{S}}=a_{p q} ; \quad \Delta=\left|\begin{array}{cccc}
a_{11}, & a_{12}, & \ldots & a_{1 n} \\
a_{21}, & a_{22}, & \ldots & a_{2 n} \\
\cdot & \cdot & \cdot & \cdot \\
\cdot & \cdot & \cdot & \cdot \\
\cdot & \cdot & \cdot & \cdot \\
a_{n 1}, & a_{n 2}, & \ldots & \cdot \\
& . & a_{n n}
\end{array}\right| ; \quad \Delta_{p q}=\frac{\partial \Delta}{\partial a_{p q}}
$$

and

$$
\frac{\partial^{k_{1}+k_{2}+\ldots+k_{n}} e^{-\frac{1}{2 \Delta} \Sigma \Sigma \Delta_{p q} w_{p} w_{q}}}{\partial w_{1}^{k_{1}} \partial w_{2}^{k_{3}} \ldots \partial w_{n}^{k_{n}}}=\mathrm{R}_{k_{1} k_{2} \ldots k_{n}}\left(w_{1}, w_{2}, \ldots w_{n}\right) e^{-\frac{1}{2 \Delta} \Sigma \Sigma \Delta p q w_{p} w_{q}}
$$

the general formula

$$
\begin{array}{r}
\frac{\sqrt{\Delta}}{(2 \pi)^{n / 2}} \int_{-\infty}^{\infty} d x_{1} \int_{-\infty}^{\infty} d x_{2} \ldots \int_{-\infty}^{\infty} d x_{n} x_{1}^{k_{1}} x_{2}^{k_{2}} \ldots x_{n}^{k_{n}} e^{-\frac{1}{2} \Sigma \Sigma \alpha_{p q} x_{p} x_{n}} \\
=(-1)^{\frac{k_{1}+k_{2}+\ldots .+k_{n}}{2}} \mathrm{R}_{k_{1} k_{2} \ldots, \ldots k_{n}}(0)
\end{array}
$$

Here $a_{p q}$ may be any real quantities, and up to the sixth order the value of the integral is obtained if in the respective equations (6) and (8) $r_{p q}$ is exchanged for $\frac{\Delta_{p q}}{\Delta}$.

\section{APPENDIX.}

In order to prove equation (2) we put, using the theorem of Fourier,

$$
\begin{aligned}
\phi\left(x_{1}, x_{2}, \ldots v_{n}\right) & =\frac{1}{(2 \pi)^{n}} \int_{-\infty}^{\infty} d w_{1} \int_{-\infty}^{\infty} d w_{2} \ldots \int_{-\infty}^{\infty} d u_{n} e^{-i \Sigma x p w_{p}} \\
& \times \int_{-\infty}^{\infty} d \lambda_{1} \int_{-\infty}^{\infty} d \lambda_{2} \ldots \int_{-\infty}^{\infty} d \lambda_{n} \phi\left(\lambda_{1}, \lambda_{2} \ldots \lambda_{n}\right) e^{i \Sigma w_{p} \lambda_{p}} .
\end{aligned}
$$


It will thus be required to show that

$$
\begin{array}{r}
\frac{1}{(2 \pi)^{n} \sqrt{\mathrm{S}}} \int_{-\infty}^{\infty} d \lambda_{1} \int_{-\infty}^{\infty} d \lambda_{2} \ldots \int_{-\infty}^{\infty} d \lambda_{n} e^{-\frac{1}{2 \mathbb{S}} \Sigma \Sigma S_{p q} \lambda_{p} \lambda_{q}+i \Sigma w_{p} \lambda_{p}} \\
=e^{-\frac{1}{2} \Sigma \Sigma r_{p q} w_{p} w_{q}}
\end{array}
$$

The evaluation of this integral will be possible if the exponent $-\frac{1}{2 \mathrm{~S}} \Sigma \Sigma \mathrm{S}_{p q} \lambda_{p} \lambda_{q}+i \Sigma w_{p} \lambda_{p}$ is reduced to the form $-\frac{1}{2 S} \sum \Sigma S_{p q}\left(\lambda_{p}+\alpha_{p}\right)\left(\lambda_{q}+\alpha_{q}\right)+\frac{1}{2 S} \sum \Sigma S_{p q} \alpha_{p} \alpha_{q}$ and the integral (1) is then equal to

$$
e^{\frac{1}{2 S} \Sigma S_{p q} x_{p} \alpha_{q}}
$$

Thus it remains only to show that

$$
\frac{1}{2 \mathrm{~S}} \Sigma \mathrm{S}_{p q} \alpha_{p} \alpha_{q}=-\frac{1}{2} \Sigma \Sigma r_{p q} w_{p} w_{q}
$$

Putting $i \lambda_{p}=\lambda_{p}{ }^{\prime}$ and $i \alpha_{p}=\alpha_{p}{ }^{\prime}$, we have

$$
\begin{aligned}
& \frac{1}{2 \mathrm{~S}} \Sigma \Sigma \mathrm{S}_{p q} \lambda_{p}{ }^{\prime} \lambda_{q}{ }^{\prime}+\Sigma w_{p} \lambda_{p}{ }^{\prime} \\
& =\frac{1}{2 \mathrm{~S}} \Sigma \Sigma \mathrm{S}_{p q}\left(\lambda_{p}{ }^{\prime}+\alpha_{p}{ }^{\prime}\right)\left(\lambda_{q}{ }^{\prime}+\alpha_{q}{ }^{\prime}\right)-\frac{1}{2 \mathrm{~S}} \Sigma \Sigma \mathrm{S}_{p q} \alpha_{p}{ }^{\prime} \alpha_{q}{ }^{\prime} \\
& =\frac{1}{2 \mathrm{~S}} \Sigma \Sigma \mathrm{S}_{p q} \lambda_{p}{ }^{\prime} \lambda_{q}{ }^{\prime}+\frac{1}{\mathrm{~S}} \Sigma \Sigma \mathrm{S}_{p q^{\prime}} \lambda_{p^{\prime}}{ }^{\prime} \alpha_{q}{ }^{\prime}
\end{aligned}
$$

Hence we have

$$
w_{s}=\frac{1}{\mathrm{~S}_{t}} \Sigma_{t} \mathrm{~S}_{s t} \alpha_{t}^{\prime}
$$

This is a system of linear equations to letermine the different $\alpha_{t}{ }^{\prime}$.

Putting

$$
\Delta=\mid \begin{aligned}
& \mathrm{S}_{s t} \\
& \mathrm{~S}
\end{aligned} ; \quad \Delta_{s t}=\frac{\partial \hat{\Lambda}}{\partial \frac{\mathrm{S}_{s t}}{\mathrm{~S}}},
$$

the solution is

$$
\alpha_{s}^{\prime}=\frac{1}{\Delta} \sum_{t} \Delta_{s t} w_{t}
$$


452 Correlation Function of any Number of Variates.

But according to well-known theorems on determinants, we have

$$
\frac{\Delta_{s t}}{\Delta}=r_{s t}
$$

and hence

For the sum

$$
\alpha_{s}^{\prime}=\sum_{t} r_{s t} w t
$$

$$
\frac{1}{2 \mathrm{~S}} \sum_{p} \sum_{q} \mathrm{~S}_{p q} \alpha_{p}^{\prime} x_{q}^{\prime}
$$

we may now write

$$
\frac{1}{2 \bar{S}} \sum_{p q} \sum_{q} S_{p q} \sum_{t} r_{p t} w_{t} \Sigma_{s} r_{s q} w_{s}
$$

Changing the order of summation, we hare this equal to

$$
\frac{1}{2 \mathrm{~S}} \sum_{t} \sum_{t} w_{s} w_{t} \sum_{p} r_{p t} \sum_{q} S_{p q} p_{s q}
$$

But

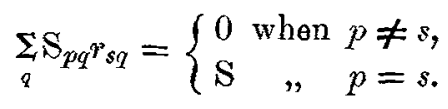

Thus the quadruple sum is equal to

$$
\frac{1}{2 \mathrm{~S}} \sum_{t} \sum_{t} w_{s} w_{t} \eta_{s t}^{2} \mathrm{~S}
$$

or

$$
\frac{1}{2} \mathrm{~S}_{p} \sum_{q} \mathbb{S}_{p q^{\alpha} \alpha^{\prime} \alpha_{q}}=\frac{1}{2} \sum_{p} \sum_{q} r_{p q} i{ }_{p} w_{q}
$$

As $\alpha_{p}{ }^{\prime}=i a_{p}$, we have finally

$$
\underset{2 \mathrm{~S}}{1} \sum_{p} \sum_{q} \mathrm{~S}_{p q} \alpha_{p} \alpha_{q}=-\frac{1}{2} \sum_{p} \sum_{q} r_{q} w_{p} w_{q} .
$$

Q. E. D. 